Original Research Paper

\title{
What is Missing in STD Screening in Hong Kong?
}

\author{
${ }^{1}$ Clement Leung-Kwok Chan, ${ }^{2}$ Wai Yee Chan and ${ }^{3}$ Christopher Hon Ki Cheng and ${ }^{1,4}$ Ping Xia \\ ${ }^{1,4}$ Women's Health and Reproductive Medicine Centre, Hong Kong \\ ${ }^{1-3}$ School of Biomedical Sciences, Faculty of Medicine, The Chinese University of Hong Kong, Hong Kong \\ ${ }^{4}$ The Johns Hopkins University School of Medicine, Baltimore, MD, USA
}

\section{Article history}

Received: 08-04-2016

Revised: 29-12-2016

Accepted: 11-01-2016

Corresponding Author:

Ping Xia

Women's Health and

Reproductive Medicine Centre,

Hong Kong and

The Johns Hopkins University

School of Medicine, Baltimore, MD, USA

Email: pxia2@jhmi.edu

\begin{abstract}
This retrospective analysis was to study the prevalence of Sexually Transmitted Diseases (STD) in a Reproductive Medical Center in Hong Kong. A total of 1190 patients were included in this study. Group 1A, couples had no symptoms but presented with subinfertility; Group 1B, the subfertile couples were positive for either Chlamydia trachomatis (CT) or Ureaplasma urealyticum (UU); Group 2, couples with symptoms were offered full STD screening including CT, UU, Mycoplasma hominis (MH), Neisseria gonorrhoea (NG), Syphilis, Herpes Simplex Virus 1 and 2. The methods of ELISA and quantitative real-time PCR were used for these analyses. Group 1A: UU detection rates in both male and female (13.84 v.s. $37.07 \%$ ) were significantly higher than that of CT (5.65 v.s. $5.57 \%$ ); Group $1 \mathrm{~B}$ and Group 2: For those who had Full STD check, UU and MH detection rates were significantly higher than that of CT $(35,13.7$ and $7.1 \%$ respectively). Over $47 \%$ of patients showed positive for one or more organism. In the subfertile couples, the UU and CT detection rates were much higher in females than those in males. Both semen and urine samples gave the same rates of infection among $\mathrm{CT}, \mathrm{UU}, \mathrm{NG}$ and $\mathrm{MH}$. $U$. urealyticum infection rate rather than Chlamydis trachomatis infection is highest in Hong Kong. The infection rate in females is higher than in males. The detection rates in semen and urine samples in males are similar.
\end{abstract}

Keywords: STD, Reproduction, Ureaplasma urealyticum, Chlamydia trachomatis, Hong Kong

\section{Introduction}

The screening for Sexually Transmitted Diseases (STD) is a routine procedure for all patients receiving fertility treatment. With an increasing demand on sperm donors and egg donors, STD screening draws more attention to both patients and professionals. In U.S., STD prevalence was 15 million (Cates, 1999) and this number continues to rise every year. To date, there are not many publications on STD in the infertility field. Such information would shed light on making guidelines by the regulatory bodies.

According to the Hong Kong guidelines for screening of potential gamete/embryo donors and recipients against infectious diseases, the following serological tests should be performed:

- HIV

- Syphilis

- Hepatitis B surface antigen (HBsAg)
- Hepatitis B core antibody (anti-HBc) (IgG and IgM)

- Hepatitis C antibody

- Antibody tests (IgG and IgM) for CMV

- Semen, urine or urethral cultures for Neisseria gonorrhoeae. Either urethral or urinary testing for Chlamydia trachomatis should be performed

$\mathrm{CT}$ is an obligate intracellular human pathogen. It is a gram-negative bacterium which appears as either coccoid or rod shape under light microscope. CT includes three human biovars: (1) Serovars Ab, B, Ba or C. They cause infection of the eyes (trachoma), which can lead to blindness. (2) Serovars D-K can cause urethritis, pelvic inflammatory disease, ectopic pregnancy, neonatal pneumonia and neonatal conjunctivitis. (3) Serovars L1, L2 and L3 which could cause lymphogranuloma venereum.

CT infections may cause chronic pelvic pain, ectopic pregnancy and infertility. Patients with CT infections are often not aware of their disease. In U.S., there are approximately 4 million people infected each year. Its 
prevalence is about 5\% (CDCP, 2009). In South Africa, the reported infection rates were higher (5.9-33\%) among general populations showing a big regional difference (Buve et al., 2001; Isibor et al., 2005; Nwankwo and Sadiq, 2014). Therefore CT has been the main target for general population screening.

Non-specific urethritis, proctitis (rectal disease with bleeding), trachoma and infertility are seen in both genders. The most commonly seen in men are prostatitis and epididymitis. Women, once infected, would suffer from cervicitis, pelvic inflammatory disease, ectopic pregnancy and acute/chronic pelvic pain. Trachoma and pulmonary infection are also seen in neonates. There are approximately 600 million people infected with Chlamydia worldwide, of which 20 million became blind. In Hong Kong, it is unclear whether there are other pathogens which need to be screened apart from the routinely screened STDs.

The aims of this study are to address three questions, namely, (1) Is CT the most prevalent organism in Hong Kong? (2) What is the relative prevalence of the various STD organisms? (3) For males, which sample is the more sensitive for STD screening, semen or urine?

\section{Materials and Methods}

This is a retro prospective study encompassing over 1190 patients, approved by the local Ethic Committee, chaired by the first author, Clement Leung-Kwok Chan. The urine and semen samples were routinely collected from the patients who visited the Women's Health and Reproductive Medical Center in Hong Kong during July 1, 2005 to June 30 2013. Patients were divided into two groups based on their presenting symptoms.

Group 1A: Female patients presented with subfertility or with vaginal discharge and male subfertility patients with discharges went through initial screening for DNA of CT ( $=1039)$ and UU $(n=1190)$ using the method of Real Time PCR.

Group 1B: If these patients were positive for the above initial testing, the couples were tested with full STD checking including CT, UU, MH, NG, syphilis (VDRL or RPR), IgG of Herpes Simplex Virus (HSV) 1 and 2.

Group 2: Full STD check was also carried out for the patients who presented with pelvic or genital infection, or pelvic pain $(n=620)$.

\section{Real-Time PCR}

Detection of $\mathrm{CT}, \mathrm{NG}, \mathrm{MH}$, UU DNA in endocervical scrapping, semen and urine samples was conducted by the methods of real-time PCR using Applied Biosystem 7500 (basedon 36\% prevalence of infection, sensitivity, specificity, PPV and NPV were $87,96,94$ and $93 \%$ respectively).

\section{Syphilis Serology Detection}

Rapid Plasma Reagin (RPR): RPR Card Test (Arlington Scientific, Inc., ASI) for Syphilis was conducted. It is a qualitative and semi-quantitative nontreponemal flocculation test for detection of reagin antibodies in human serum and plasma.

\section{Enzyme-Linked Immunosorbent Assay (ELISA)}

Antibodies against HSV type 1 and HSV type 2 were analyzed by ELISA with $100 \%$ sensitivity and specificity.

The Chlamydia antigens were analyzed by ELISA. The antibodies against Chlamydia antigens were detected by Direct Fluorescent Antibody Test (DFA).

\section{Chlamydia Cell Culture}

Suspected Chlamydia samples were cultured in a vial of cells in the laboratory. The pathogen infected cells, after $48 \mathrm{~h}$ (up to 2 days), were stained and viewed under a fluorescent light microscope.

The data were analysed by Chi Square Test.

\section{Results}

Results of Group 1A were tabulated in the Table 1. The infection rate for CT was similar in both female and male patients (5.57 and 5.65\%). Both female and male patients had higher detection rates of UU compared with those of CT (37.07 v.s. $5.57 \%, \mathrm{P}<0.001$ in females; 13.84 v.s. $5.65 \%, \mathrm{P}=0.006$ in males). Also, detection rate for UU was significantly higher in females (37.07\%) compared to males $(13.84 \%, \mathrm{P}<0.001)$.

Among the full STD check (Group 1B and Group 2, $\mathrm{n}=620), 52 \%$ of the patients were negative, whereas UU detection rate was highest $(27.9 \%)$ in all categories detected (Table 2).

Table 3 showed detection rates in those with full STD check in relation to the presenting symptoms. There is no difference among the diagnoses. However, the detection rate for HSV1was the highest $(74.5 \%, \mathrm{P}=0.015)$, followed by the second highest of UU infection $(35.5 \%$ $\mathrm{P}<0.001$ ) (Table 3).

Table 1. Initial screening of CT and UU for patient Group 1A

\begin{tabular}{llll}
\hline & Chlamydia trachomatis & Ureaplasma urealyticum & Pvalue \\
\hline Female & $48 / 862(5.57 \%)$ & $334 / 901(37.07 \%)$ & $<0.001$ \\
Male & $10 / 177(5.65 \%)$ & $40 / 289(13.84 \%)$ & $<0.01$ \\
Pvalue & $>0.05$ & $<0.001$ & \\
No. of cases & 1039 & 1190 & \\
\hline
\end{tabular}


Table 2. Full STD screening

\begin{tabular}{lc}
\hline Categories & Frequency \\
\hline CT & $22 / 620(3.55 \%)$ \\
NG & $1 / 620(0.16 \%)$ \\
UU & $173 / 620(27.90 \%)$ \\
MH & $47 / 620(7.58 \%)$ \\
CT+UU & $13 / 620(2.10 \%)$ \\
CT+ & $6 / 620(0.97 \%)$ \\
NG+UU & $4 / 620(0.65 \%)$ \\
UU+MH & $27 / 620(4.35 \%)$ \\
CT+UU+MH & $4 / 620(0.65 \%)$ \\
All negative & $323 / 620(52.10 \%)$ \\
\hline
\end{tabular}

Table 3. Association of different infections and various diagnoses

\begin{tabular}{|c|c|c|c|c|c|c|c|}
\hline & $\mathrm{CC}$ & NG & $\mathrm{MH}$ & UU & VDRL/RPR & HSV type 2 IgG & HSV type $1 \mathrm{IgG}$ \\
\hline Infection frequency & $10 / 81(12.35 \%)$ & $0 / 81(0.00 \%)$ & $10 / 81(12.35 \%)$ & 9/81 (11.11\%) & $0 / 81(0.00 \%)$ & $10 / 81(12.35 \%)$ & $58 / 81(71.60 \%)$ \\
\hline Pelvic discomfort & $10 / 92(10.87 \%)$ & $1 / 92(1.09 \%)$ & $13 / 92(14.13 \%)$ & $36 / 92(39.13 \%)$ & $1 / 92(1.09 \%)$ & $21 / 92(22.83 \%)$ & $78 / 92(84.78 \%)$ \\
\hline Subfertility & $19 / 343(5.54 \%)$ & $4 / 343(1.17 \%)$ & $46 / 343(13.41 \%)$ & $139 / 343(40.52 \%)$ & $3 / 343(0.87 \%)$ & $67 / 343(19.53 \%)$ & $247 / 343(72.01 \%)$ \\
\hline Vaginal discharge & $4 / 63(6.35 \%)$ & $0 / 63(0.00 \%)$ & $10 / 63(15.87 \%)$ & $26 / 63(41.27 \%)$ & $0 / 63(0.00 \%)$ & $10 / 63(15.87 \%)$ & $53 / 63(84.13 \%)$ \\
\hline Unclassified & $1 / 41(2.44 \%)$ & $0 / 41(0.00 \%)$ & $6 / 41(14.63 \%)$ & $10 / 41(24.39 \%)$ & $0 / 41(0.00 \%)$ & $10 / 41(24.39 \%)$ & $26 / 41(63.41 \%)$ \\
\hline Total & $44 / 620(7.1 \%)$ & $5 / 620(0.8 \%)$ & $85 / 620(13.7 \%)$ & $220 / 620(35.5 \%)$ & $4 / 620(0.64 \%)$ & $118 / 620(19 \%)$ & $462 / 620(74.5 \%)$ \\
\hline Pvalue & $>0.05$ & $>0.05$ & $>0.05$ & $<0.001$ & $>0.05$ & $>0.05$ & $<0.05$ \\
\hline
\end{tabular}

Table 4. Association of different infections and gender

\begin{tabular}{|c|c|c|c|c|c|c|c|}
\hline & $\mathrm{CT}$ & NG & $\mathrm{MH}$ & UU & VDRL/RPR & HSV type 2 IgG & HSV type $1 \mathrm{IgG}$ \\
\hline Female & $14 / 127(11.03 \%)$ & $3 / 127(2.36 \%)$ & $23 / 127(18.11 \%)$ & $83 / 127(65.35 \%)$ & $1 / 127(0.79 \%)$ & $29 / 127(22.83 \%)$ & $100 / 127(78.74 \%)$ \\
\hline Male & $4 / 127(3.15 \%)$ & $0 / 127(0 \%)$ & $16 / 127(12.60 \%)$ & $31 / 127(24.41 \%)$ & $1 / 127(0.79 \%)$ & $18 / 127(14.17 \%)$ & $86 / 127(67.71 \%)$ \\
\hline Pvalue & $\mathrm{P}=0.014$ & $>0.05$ & $>0.05$ & $<0.001$ & $>0.05$ & $>0.05$ & $\mathrm{P}=0.047$ \\
\hline
\end{tabular}

The frequency of infections between female and male $(\mathrm{n}=127)$ was summarized in Table 4 . CC infection rate was higher in females than that in males (11.03 v.s. $3.15 \% ; \mathrm{P}=0.014)$. UU infection was also higher in females (65.35 v.s. $24.41 \%$; $\mathrm{P}<0.001)$ (Table 4).

When comparing the infection chances in semen and urine samples, there is no difference in both approaches in collecting samples (data not included). In another words, the patient can have their semen or urine checked for the presence of these organisms.

\section{Discussion}

This study, for the first time in Hong Kong, revealed that UU infection rates in both male and female subfertile patients were higher than those of CT infection; Among the full STD screening, UU still represented the highest rate of infection; Among CT, NG, $\mathrm{MH}$ and $\mathrm{UU}, \mathrm{MH}$ showed significantly higher detection rates compared to that of $\mathrm{CT}$. There was no significant difference among patients with different presenting symptoms; in the subset of 127 subfertility couples, the UU and CT detection rates in female were significantly higher than the those of the male partners; lastly, both semen and urine samples showed the same rate of infections.

Many studies in the past two decades have shown great regional difference worldwide. A study reported that in Israel when the patients showed U. Urealyticum positive, the chance of Condyloma Acuminatum was higher (Zvulunov et al., 2000). Our results further demonstrated $U$. Urealyticum and HSV type 1 should be included in the routine STD screening in Hong Kong, especially for the subfertile patients. As early as in 1997, a large scale study including 41,980 patients in Vienna, Austria, who suffered from infectious VeneroDermatological Diseases, Mycoplasma Hominis and Ureaplasma urealyticum, were detected more often in the vaginal fluid that in the male urethra (Koch et al., 1997).

Ureaplasma have two species-urealyticum and parvum. Ureaplasma urealyticum is a bacterium belonging to the family of Mycoplasmataceae. $U$. parvum serovar 3/14 and T960 biovar were found to be significantly associated with symptomatic patients, while on the contrary, $U$. parvum serovar 6 was significantly correlated with asymptomatic women and normal vaginal flora (De Francesco et al., 2009). Ureaplasmaparvum is more prevalent than Ureaplasma urealyticum in specimens from infertile men (Abusarahet et al., 2013). U. urealyticum is part of the normal genital flora of both men and women. Indeed its infection was reported not affecting semen quality (Wang et al., 2006; Gdoura et al., 2007). However, U. Urealyticum infection is closely related to reduction in the pregnancy rate of IVF patients, possibly because of its infection at the endometrial level owing to the fact that fertilization rate and the total number of eggs retrieved was not affected (Montagut et al., 1991).

$\mathrm{MH}$, UU were thought for several decades among experts in genital tract infectious disease to be part of 
normal flora of sexually active women. However, it is not known that both may cause chorioamnionitis, salpingitis, acterialvaginosis and postpartum endometritis and therefore they should no longer be considered as commensals (Larsen and Hwang, 2010). In addition, it was reported that Ureaplasma was detected positive in $50 \%$ preterm babies in one or more compartments (i.e., Respiratory, blood and/or cerebrospinal fluid) (Viscardi, 2014).

Moreover, Potts et al. (2000) reported seminal Reactive Oxygen Species (ROS) levels were elevated among patients infected with $U$. Urealyticum. These authors suggested that ROS induces lipid peroxidation, which reduces membrane fluidity and sperm fertilization capability, possibly explaining how $U$. Urealyticum impairs sperm function. The level of Zinc and Selenium in the seminal fluid is decreased in Urealyticum infected patients (Han et al., 2003). Based on the characteristics, U. urealyticum has two genotypes-biovarI (serovars 1,3 , 6 and 14) and biovar II (serovars 2, 4, 5 and 7-13) (Povlsen et al., 1998). Biovar II is more likely related to male infertility (Zhang et al., 2014a). This work was conducted in Southern China. Whether it is also the same in Hong Kong needs to be further studied.

The tissue sources and methods of detection for STD pathogens resulted in a variety of outcomes. The tests of using menstrual tissues were proven to be sensitive, allowing early detection and early treatment of a condition that can otherwise lead to serious consequences such as tubal obstruction, pelvic inflammatory disease, ectopic pregnancy, spontaneous abortions and unexplained infertility (Michou et al., 2014). Detection of Neisseria Gonorrhoeae in pregnant women in Iran showed that endocervical swab specimens should be detected by both Nucleic Acid Amplification Tests and culture method to limit false negative samples (Hassanzadeh et al., 2013). Recently a novel method of using bead-based multiplex Sexually Transmitted Infection Profiling was reported (Schmitt et al., 2014). This method specifically detects Chlamydia trachomatis, HSV type 1 and 2, Treponema pallidum, Trichomonas vaginalis, Neisseria gonorrhoeae, Mycoplasma, M. genitalium, $M$. hominis, $M$. pneumonia, $M$. spermatophilum, Ureaplasma urealyticum and U. Parvum and quantifies bacterial vaginosis-associated Atopobium vaginae and Gardnerella vaginalis as well as three Candida species and normal genital flora-associated Lactobacillus species. Moreover, a Multilocus sequence typing scheme based on four housekeeping genes ( $f t s H$, $r p L 22, v a l S, t h r S$ ) is more adequate for investigations of molecular epidemiology and population structure with highly discriminating capacity (Zhang et al., 2014b).

Our results concluded both semen and urine samples presented similar detection rates for C. trachomatis, $N$. gonorrhoeae, M. hominis, U. urealyticum, syphilis and
HSV type 1 and 2. A similar finding was reported that there is no difference between semen samples and First Void Urine (FVU) samples in detecting C. trachomatis, as well as $U$. Urealyticum in infertile patients in Jordan (Abusarahet et al., 2013) whereas N. Gonorrhoeae was found to be present in semen rather than FVU which was different from our result.

As far as treatments are concerned, the Ureaplasma are tested against azithromycin, josamycin, ofloxacin and doxycycline. Resistance to macrolides, tetracyclines and fluoroquinolones has been reported (Kokkayil and Dhawan, 2015). Patients with infection of $U$. urealyticum have been associated with low birth weight and premature delivery (Kafetzis et al., 2004). Therefore appropriate treatments prior to conception are essential in achieving healthy pregnancies.

\section{Conclusion}

(1) Ureaplasma urealyticum infection rates in both male and female were significantly higher than those of Chlamydia trachomatis infection; (2) Among the full STD check $U$. urealyticum still represents the highest rate of infection; (3) U. urealyticum exhibited the second highest infection among all diagnoses including pelvic discomfort, subfertility, vaginal discharge and unclassified diagnoses; (4) In female patients, U. urealyticum infection rate is much higher than that in the males; (5) Both semen and urine samples gave the same rate of infection among C. trachomatis, N. gonorrhoeae, M. hominis, $U$. urealyticum, Syphilis and HSV type 1 and 2.

\section{Acknowledgement}

We thank the nurses and clinical staff at the Women's Health and Reproductive Medicine Centre for assisting the patient data collection. We are also imensely grateful to Dr. Yionghuak Chan at the National University of Singapore for assistance of statistic analysis.

\section{Funding Information}

This work was supported by Professor Clement Leung-Kwok Chan's Private Funding.

\section{Author's Contributions}

Clement Leung-Kwok Chan: Initiated project; Recruiting patients; Data collection; Experimental Design and contribution to the key ideas.

Wai Yee Chan: Manuscript revision; Experimental design and Critical Review for significant intellectual content.

Christopher Hon Ki Cheng: Manuscript revision; Data analysis and Critical Review for significant intellectual content. 
Ping Xia: Interpretation of the data; manuscript draft; Critical Review for significant intellectual content and Manuscript submission and revisions.

\section{Ethics}

No ethical issues should arise from this publication.

\section{References}

Cates, W.J., 1999. Estimates of the incidence and prevalence of sexually transmitted diseases in the United States. Sex. Transm. Dis., 26: S2-7. DOI: 10.1097/00007435-199904001-00002

CDCP, 2009. Chlamydia screening among sexually active young female enrollees of health plans-United States, 2000-2007. Morbidity Mortality Weekly Report, 58: 362-365.

Buve, A., H.A. Weiss, M. Laga, E. Van Dyck and R. Musonda et al., 2001. The epidemiology of gonorrhoea, chlamydial infection and syphilis in four African cities. AIDS, 15: 579-588. PMID: 11686469

Isibor, J.O., D. Ugbomoiko, G.O. Nwobu, A.O. Ekundayo and I.B. Eweani et al., 2005. Detection of Chlamydia antigen in cervical specimens from antenatal clinic attendees in Benin City, Nigeria. Am. J. Clin. Exp. Microbiol., 6: 208-211.

Nwankwo, E.O. and M.N. Sadiq, 2014. Prevalence of Chlamydia trachomatis infection among patients attending infertility and Sexually Transmitted Diseases clinic (STD) in Kano, North Western Nigeria. Afr. Health Sci., 14: 672-678.

DOI: 10.4314/ahs.v14i3.24

Zvulunov, A., E. Medvedovsky, A. Biton, S. Horowitz and D. Vardy, 2000. Association of Ureaplasma urealyticum colonization in male urethra and Condyloma acuminatum. Isr. Med. Assoc. J., 2: 580-582. PMID: 10979348

Koch, A., A. Bilina, L. Teodorowicz and A. Stary, 1997. Mycoplasma hominis and Ureaplasma urealyticum in patients with sexually transmitted diseases. Wien Klin. Wochenschr., 109: 584-589. PMID: 9286064

De Francesco, M.A., R. Negrini, G. Pinsi, L. Peroni and N. Manca, 2009. Detection of Ureaplasma biovars and polymerase chain reaction-based subtyping of Ureaplasma parvum in women with or without symptoms of genital infections. Eur. J. Clin. Microbiol. Infect. Dis., 28: 641-646.

DOI: $10.1007 / \mathrm{s} 10096-008-0687-\mathrm{z}$

Abusarahet, E.A., Z.M. Awwad, E. Charvalos and A.A. Shehabi, 2013. Molecular detection of potential sexually transmitted pathogens in semen and urine specimens of infertile and fertile males. Diagn. Microbiol. Infect. Dis., 77: 283-286.

DOI: 10.1016/j.diagmicrobio.2013.05.018
Wang, Y., C.L. Liang, J.Q. Wu, C. Xu and S.X. Qin et al., 2006. Do Ureaplasma urealyticum infections in the genital tract affect semen quality? Asian J. Androl., 8: 562-568. DOI: 10.1111/j.1745-7262.2006.00190.x

Gdoura, R., W. Kchaou, C. Chaari, A. Znazen and L. Keskes et al., 2007. Ureaplasma urealyticum, Ureaplasma parvum, Mycoplasma hominis and Mycoplasma genitalium infections and semen quality of infertile men. BMC Infect. Dis., 7: 129-137. DOI: $10.1186 / 1471-2334-7-129$

Montagut, J.M., S. Leprêtre, J. Degoy and M. Rousseau, 1991. Ureaplasma in semen and IVF. Hum. Reprod, 6: 727-729. PMID: 1939557

Larsen, B. and J. Hwang, 2010. Mycoplasma, Ureaplasma and adverse pregnancy outcomes: A fresh look. Infect. Dis. Obstet. Gynecol. DOI: 10.1155/2010/521921

Viscardi, R.M., 2014. Ureaplasma species: Role in neonatal morbidities and outcomes. Arch. Dis. Child Fetal. Neonatal Edition, 99: F87-F92. DOI: 10.1136/archdischild-2012-303351

Potts, J.M., R. Sharma, F. Pasqualotto, D. Nelson and G. Hall et al., 2000. Association of Ureaplasma urealyticum with abnormal reactive oxygen species levels and absence of leukocytospermia. J. Urol., 163: 1775-1778. DOI: 10.1016/S0022-5347(05)67540-4

Han, X.D., Y. Wang and J.X. Chen, 2003. A comparative study on interrelations among microelements, infection of Ureaplasma urealyticum and male infertility. Arch. Androl., 49: 265-269. PMID: 12851028

Povlsen, K., J.S. Jensen and I. Lind, 1998. Detection of Ureaplasma urealyticum by PCR and biovar determination by liquid hybridization. J. Clin. Microbiol., 36: 3211-3216. PMID: 9774567

Zhang, Q.X., Y.X. Xiao, W.D. Zhuang, B.Z. Cheng and L. Zheng et al., 2014a. Effects of biovar I and biovar II of ureaplasma urealyticum on sperm parameters, lipid peroxidation and deoxyribonucleic acid damage in male infertility. Urology, 84: 87-92. PMID: 24976225

Michou, I.V., P. Constantoulakis, K. Makarounis, G. Georgoulias and V. Kapetanios et al., 2014. Molecular investigation of menstrual tissue for the presence of Chlamydia trachomatis, Ureaplasma urealyticum and Mycoplasma hominis collected by women with a history of infertility. J. Obstet. Gynaecol. Res., 40: 237-242. PMID: 24118383

Hassanzadeh, P., J. Mardaneh and M. Motamedifar, 2013. Conventional agar-based culture method and Nucleic Acid Amplification Test (NAAT) of the cppB gene for detection of neisseria gonorrhea in pregnant women endocervical swab specimens. Iran Red. Crescent J., 15: 207-211. DOI: 10.5812/ircmj.3726

Schmitt, M., C. Depuydt, M. Stalpaert and M. Pawlita, 2014. Bead-based multiplex sexually transmitted infection profiling. J. Infect., 69: 123-133. DOI: 10.1016/j.jinf.2014.04.006 
Zhang, J., Y. Kong, Y. Feng, J. Huang and T. Song et al., 2014b. Development of a multilocus sequence typing scheme for Ureaplasma. Eur. J. Clin. Microbiol. Infect. Dis., 33: 537-544.

DOI: $10.1007 / \mathrm{s} 10096-013-1981-\mathrm{y}$

Kokkayil, P. and B. Dhawan, 2015. Ureaplasma: Current perspectives. Ind. J. Microbiol., 33: 205-214.

DOI: $10.4103 / 0255-0857.154850$
Kafetzis, D.A., C.L. Skevaki, V. Skouteri, S. Gavrili and K. Peppa et al., 2004. Maternal genital colonization with Ureaplasma urealyticum promotes preterm delivery: Association of the respiratory colonization of premature infants with chronic lung disease and increased mortality. Clin. Infect. Dis., 39: 1113-1122. DOI: 10.1086/424505 